\title{
Magnetresonanz-geführter fokussierter Ultraschall zur Myombehandlung - Ergebnisse des ersten radiologisch-gynäkologischen Expertentreffens
}

Autoren

Institute
A. Beck' ${ }^{1}$ M. David ${ }^{2}$, T. Kröncke ${ }^{1}$

Klinik für Strahlenheilkunde und Institut für Radiologie, Charité Universitätsmedizin Berlin

2 Klinik für Gynäkologie, Charité Universitätsmedizin Berlin

\section{Bibliografie}

Dol http://dx.doi.org/

10.1055/s-0033-1335338

Online-Publikation: 9.4.2013

Fortschr Röntgenstr 2013; 185:

464-466 @ Georg Thieme

Verlag KG Stuttgart · New York . ISSN 1438-9029

\section{Korrespondenzadresse \\ Dr. med. Alexander Beck}

Klinik für Strahlenheilkunde und Institut für Radiologie, Charité Universitätsmedizin Berlin Campus Virchow-Klinikum Augustenburger Platz 1 13353 Berlin

Prof. Dr. med. Matthias David Klinik für Gynäkologie, Charité Universitätsmedizin Berlin Campus Virchow-Klinikum Augustenburger Platz1 13353 Berlin

\section{PD Dr. med. Thomas Kröncke,}

\section{MBA}

Klinik für Strahlenheilkunde und Institut für Radiologie, Charité Universitätsmedizin Berlin Campus Charité Mitte

Charitéplatz 1

10117 Berlin

Tel.: +49/30/4 50-62 7394

Fax: +49/30/4 50-7-62 7394

thomas.kroencke@charite.de

\section{Präambel}

$\nabla$

Die Myombehandlung mit der Technik des Magnetresonanz-geführten fokussierten Ultraschalls (MRgFUS; Syn.: HIFU = hochintensiver fokussierter Ultraschall) ist ein thermoablatives Verfahren, bei dem durch fokussierten Ultraschall unter ständiger MR-tomografischer Kontrolle in einzelnen kleinen Volumenschritten (sogenannte Sonifikationen, Syn.: Sonikationen) das zu behandelnde Gewebe soweit erhitzt wird, bis eine vollständige Denaturierung des geplanten Myomvolumens erreicht ist. Nach erfolgter Thermoablation zeigt sich in der Erfolgskontrolle eine fehlende Kontrastmittelaufnahme des behandelten Gewebes (entspr. npv= non-perfused volume).

Das MRgFUS-Verfahren ist organerhaltend, nicht invasiv und kann ambulant durchgeführt werden. Die Behandlungsmethode ist relativ neu und wird derzeit nur von wenigen spezialisierten Einrichtungen angeboten.

Ziel der MRgFUS-Therapie ist die Verminderung oder Beseitigung myombedingter Beschwerden bei den betroffenen Frauen. Mit der Ultraschallbehandlung kann eine Myomschrumpfung erreicht werden. Eine vollständige Rückbildung des Myoms ist eher nicht zu erwarten.

Zwischen den Fachdisziplinen Frauenheilkunde und Radiologie besteht Einigkeit darüber, dass die Indikationsstellung zur notwendigen Therapie eines Uterus myomatosus nach fachärztlicher Untersuchung und Beratung durch eine Gynäkologin/ einen Gynäkologen erfolgen soll. Eine umfassende und vollständige Beratung über die Behandlungsmöglichkeiten bei symptomatischem Uterus myomatosus schließt neben den medikamentösen und operativen Behandlungsoptionen auch die beiden nicht operativen Therapiemöglichkeiten Uterusarterienembolisation (UAE) und MRgFUS ein. Die Entscheidung für oder gegen eine Therapiealterna- tive sollte unter Berücksichtigung des Patientinnenwunschs und in Kenntnis der Therapiealternativen, ihrer Erfolgschancen und Grenzen sowie typischer Nebenwirkungen und möglicher Komplikationen getroffen werden (informed consent). Mit der Möglichkeit der MRgFUS-Behandlung ist auch in Deutschland, Österreich und der Schweiz ein Therapieverfahren für Patientinnen mit myombedingten Beschwerden vorhanden, das eine weitere Therapieindividualisierung beim Uterus myomatosus ermöglicht.

\section{Ziel des Konsensustreffens}

$\nabla$

Intention dieses ersten Konsensustreffens war die Bewertung und Einordnung des MRgFUS-Verfahrens in das Therapiespektrum bei der Myombehandlung. Die 13 Teilnehmerinnen und Teilnehmer des radiologisch-gynäkologischen Expertentreffens haben unter Berücksichtigung der vorhandenen aktuellen Literatur und eigener Erfahrungen nach ausführlicher Diskussion einen Konsens zwischen den beiden beteiligten Fachrichtungen gefunden.

Die Expertenrunde war sich bewusst, dass hier über die Möglichkeiten und Grenzen eines radiologischen Therapieverfahrens zusammen mit Fachleuten der Gynäkologie diskutiert wurde, die das Verfahren selber nicht durchführen, die aber über Expertise und Erfahrung mit der Diagnostik sowie der medikamentösen und operativen Behandlung von Erkrankungen des weiblichen Genitales verfügen.

Die aus 8 Radiologen und 5 Gynäkologen zusammengesetzte Expertengruppe, die sich am 19. Januar 2013 in Berlin zum ersten radiologischgynäkologischen Konsensustreffen zur MRgFUSTherapie versammelte, gehörten auch Radiologen aus der Schweiz und aus Österreich an.

Die Gruppe verabschiedete nach ausführlicher, teilweise kontroverser Diskussion im Konsens die 
nachfolgenden Empfehlungen. Das Konsensuspapier wird von den am Ende der Arbeit aufgeführten Gynäkologen und Radiologen getragen. Es spiegelt den derzeitigen Wissensstand wider.

\section{Strukturelle Voraussetzungen zur Durchführung der MRgFUS-Therapie $\nabla$}

Die MRgFUS-Therapie sollte nur an Kliniken durchgeführt werden, die die nötige Expertise und Erfahrung zur Durchführung der MRgFUS-Therapie haben. Dies beinhaltet auch das konservative und ggfs. operative Management von Nebenwirkungen und Komplikationen. Es sollten außerdem Möglichkeiten zur Einleitung einer postinterventionellen Schmerztherapie gegeben sein.

\section{Notwendige Untersuchungen vor einer MRgFUS-Therapie}

Basis der Therapiefestlegung ist die fachärztlich-gynäkologische Untersuchung inkl. vaginalem und/oder abdominalem Ultraschall (in Abhängigkeit von der Größe des Uterus myomatosus). Notwendige Voraussetzung ist die Erstellung einer MRT mit Kontrastmittel (KM), möglichst in Bauchlage, da sich nur in der MRT die genaue Lage der umliegenden Organe, insbesondere Darmschlingen, ausreichend darstellen lassen. Die KM-Darstellung dient auch der Einschätzung, ob und in welchem Maße das Myom perfundiert ist.

Vor jeder MRgFUS muss die Indikation zur Hysteroskopie und fraktionierten Abrasio in Abhängigkeit vom Blutungsmuster und der Endometriumdicke und -struktur kritisch geprüft werden. Es sollte ein nicht länger als 12 Monate zurückliegender, unauffälliger zytologischer Abstrichbefund von der Cervix uteri vorliegen.

\section{Indikationen für eine MRgFUS-Therapie \\ $\nabla$}

Indikation für eine MRgFUS-Behandlung ist ein symptomatischer Uterus myomatosus.

Voraussetzung ist ein Uterus myomatosus, bei dem die anatomische Lage der Myome einen sicheren Zugang für den MRgFUS ermöglicht. Eine Anzahl von mehr als 5 Myomen erschwert die Behandlung. Bei Myomen mit einem Durchmesser von mehr als $10 \mathrm{~cm}$ ist die MRgFUS-Therapie aufgrund des großen Myomvolumens und der damit einhergehenden langen Behandlungszeit ebenfalls kritisch zu indizieren.

Die MRgFUS stellt eine Alternative zum operativen und medikamentösen Vorgehen sowie zur UAE dar. Grundlage der Therapieentscheidung sollte die Zielsetzung der Behandlung und der Therapiewunsch der Patientin sein. Der MRgFUS stellt bei gegebener technischer Durchführbarkeit eine gute Option für Patientinnen mit Wunsch nach einer möglichst gering invasiven Behandlung dar.

\section{Erfolgskriterien für die MRgFUS-Therapie \\ $\nabla$}

Als Therapieerfolg nach MRgFUS-Behandlung wird die Besserung oder das Verschwinden myombedingter Beschwerden angesehen. Eine Volumenreduktion wird angestrebt, gilt aber als sekundäres Therapieziel.

\section{Kontraindikationen für eine MRgFUS-Therapie}

$\nabla$

V.a. Malignom (absolut)

- Schwangerschaft (absolut)

- akuter entzündlicher Prozess (absolut)

- Uterus myomatosus mit mehr als 5 Myomen (relativ, Einzelfallentscheidung)

- Uterusmyome mit einem Durchmesser über $10 \mathrm{~cm}$ (relativ)

- kein ausreichendes Schallfenster zur Behandlung erreichbar (z.B.: Darmüberlagerungen, große Narben im Schallfenster, sehr dorsale Lage des Myoms) (relativ)

- subserös-gestielte Myome (relativ)

- Myomlage in der Hinterwand bzw. nahe am Os sacrum (relativ)

- allgemeine Kontraindikationen gegenüber MR-Kontrastmitteln (relativ)

- relative und absolute MRT-Kontraindikationen

\section{MRgFUS-Therapie bei Patientinnen mit Kinderwunsch}

Bisherige Fallberichte zeigen, dass eine Schwangerschaft nach MRgFUS möglich ist und diese erfolgreich ausgetragen werden kann, aber es liegen noch keine ausreichenden Daten für eine Empfehlung vor.

\section{Nebenwirkungen/Komplikationen der MRgFUS-Therapie}

$\nabla$

Relevante Nebenwirkungen und Komplikationen nach Durchführung einer MRgFUS-Behandlung sind selten:

- Schmerzen während der Behandlung (meist gering und kurz anhaltend)

- (leichte) Verbrennung der Haut

- geringe Entzündung des Unterhautfettgewebes und der Muskulatur der Bauchdecke

- Parästhesie des Beines aufgrund von Nervenreizung oder -schädigungen

- tiefe Beinvenenthrombose (sehr selten)

- Darmperforation (extrem selten)

Nach der Therapie ist ein vaginaler Abgang von Gewebsteilen möglich. Es kann zu verstärkten und/oder unregelmäßigen Blutungen bis zu ca. 3 Monate nach der Behandlung kommen.

\section{Nachuntersuchung nach MRgFUS-Therapie \\ $\nabla$}

Bei Beschwerderückgang wird eine fachärztliche Nachuntersuchung ca. 6 Monaten nach MRgFUS (z.B. mittels Sonografie) empfohlen. Bei Beschwerdepersistenz und/oder Komplikationen sollte dies früher erfolgen; dann ist auch die MRT-Kontrolle großzügig durchzuführen. Bei Auffälligkeiten in der Bildgebung ist eine histologische Abklärung anzustreben.

\section{Ausblick \\ $\nabla$}

Es ist geplant, in etwa 2 Jahren unter Berücksichtigung der dann vorliegenden Daten und Erfahrungen die Empfehlung zur MRgFUS-Therapie von Myomen zu überarbeiten. 


\section{Teilnehmer des Konsensustreffens}

Prof. Dr. med. Christoph A. Binkert/Winterthur $(\mathrm{CH})$ PD Dr. med. Michael Bohlmann/Lübeck Prof. Dr. med. Gerlinde Debus/Dachau PD Dr. med. Peter Hunold/Lübeck Prof. Dr. med. Augustinus L. Jakob/Zürich (CH) Dr. med. Matthias Matzko/Dachau Dr. med. Göntje Peters/Kiel Prof. Dr. Dr. Thomas Rabe/Heidelberg Prim. Univ.-Prof. Dr. Siegfried Thurnher/Wien (AT) Prof. Dr. med. Dierk Vorwerk/Ingolstadt

\section{Beteiligte Fachgesellschaften und Arbeitsgemeinschaften}

$\nabla$

AGE, Arbeitsgemeinschaft Gynäkologische Endoskopie DeGIR, Deutsche Gesellschaft für Interventionelle Radiologie DGGEF, Arbeitsgemeinschaft Gynäkologische Endokrinologie und Fortpflanzungsmedizin e.V.

DGGG, Deutsche Gesellschaft für Gynäkologie und Geburtshilfe DRG, Deutsche Röntgengesellschaft

ÖGIR, Österreichische Gesellschaft für Interventionelle Radiologie SSCVIR, Swiss Society of Cardiovascular and Interventional Radiology 